\title{
Hadronic Models of the Fermi Bubbles: Future Perspectives
}

\author{
Soebur Razzaque ${ }^{1, t, * \text { (D) }}$ and Lili Yang $2,1, t, *$ (D) \\ 1 Department of Physics, University of Johannesburg, PO Box 524, Auckland Park 2006, South Africa \\ 2 Center for Astrophysics and Cosmology, University of Nova Gorica, Vipavska 13, SI-5000 Nova Gorica, \\ Slovenia \\ * $\quad$ Correspondence: srazzaque@uj.ac.za (S.R.); lili.yang@ung.si (L.Y.) \\ + These authors contributed equally to this work.
}

Received: 15 February 2018; Accepted: 8 April 2018; Published: 12 April 2018

\begin{abstract}
The origin of sub-TeV gamma rays detected by Fermi-LAT from the Fermi bubbles at the Galactic center is still unknown. In a hadronic model, acceleration of protons and/or nuclei and their subsequent interactions with gas in the bubble volume can produce observed gamma rays. Such interactions naturally produce high-energy neutrinos, and a detection of those can discriminate between a hadronic and a leptonic origin of gamma rays. Additional constraints on the Fermi bubbles gamma-ray flux in the $\mathrm{TeV}$ range from recent $\mathrm{HAWC}$ observations restrict hadronic model parameters, which in turn disfavor Fermi bubbles as the origin of a large fraction of neutrino events detected by IceCube along the bubble directions. We revisit our hadronic model and discuss future constraints on parameters from observations in very high-energy gamma rays and neutrinos.
\end{abstract}

Keywords: Fermi bubbles; hadronic model; gamma rays; neutrinos

\section{Introduction}

The Fermi bubbles (FB), discovered in the gamma-ray data of the Fermi Large Area Telescope (LAT), are two giant structures extending up to 55 degrees ( $\sim 9 \mathrm{kpc}$ ) above and below the Galactic center (GC) [1-4]. The associated multi-wavelength observations in microwaves (WMAP haze) [5,6], X-ray [7,8], and polarized radio waves [9] provide comprehensive information for studying the physical origin of the bubbles. Several theoretical models have been proposed to explain the morphology and spectral properties of detected gamma rays, typically classified as hadronic and leptonic models. Both mechanisms can reproduce the observed hard spectrum, sharp edges, and uniform emission at latitudes $|\mathrm{b}|>10^{\circ}$. In the hadronic models, gamma rays are generated by inelastic collisions of accelerated cosmic rays on thermal nuclei in the bubble gas [3,10-12]. While in the leptonic models, inverse Compton scattering of relativistic electrons on optical and UV photons produces the gamma rays $[2,3,13,14]$. The true origin of gamma rays, whether hadronic or leptonic, has profound implications for the history of star-formation activities in the central region of the Milky Way and/or the activity of the super-massive black hole at the center, as well as particle acceleration.

In hadronic models, $\mathrm{TeV}$ to $\mathrm{PeV}$ neutrinos, resulting from charged pion/kaon decays, as counterparts of $\mathrm{GeV}$ gamma rays, resulting mostly from neutral pion decays, should exist $[10,12]$. Detection of these high energy neutrinos can serve as one of the major discriminators between the hadronic and leptonic models. It has been pointed out in the past that a fraction of the high-energy astrophysical neutrinos detected by IceCube [15] could originate from the FB in hadronic models [16-20]. The corresponding neutrino flux from the bubbles is consistent with the hadronic flux model that reproduce FB gamma ray data. If that is the case, the bubbles could be the first multi-messenger study source in our Milky Way [20,21]. Recently, researchers using the High Altitude 
Water Cherenkov (HAWC) telescope reported an analysis of gamma-ray data from the high-latitude $\left(b>6^{\circ}\right)$ northern FB region collected over 290 days [22] and found no evident excess above $\sim 1 \mathrm{TeV}$. The HAWC upper limits are consistent with the gamma-ray flux from Fermi-LAT but constrained our previous "neutrino-inspired" hadronic models that predict a high gamma-ray flux level in the $\geq 1 \mathrm{TeV}$ range from the $\mathrm{FB}$ region [21].

The morphology of the FB gamma-ray emission, however, is not fully defined, especially toward the GC region, which has also not been clearly observed at other wavelengths. In the latest Fermi-LAT analysis [23], it was found that the spectra of the low-latitude $\left(|b|<10^{\circ}\right)$ and high-latitude $\left(|b|>10^{\circ}\right)$ bubbles are similar in the $100 \mathrm{MeV}-100 \mathrm{GeV}$ energy range, but different at energies above $100 \mathrm{GeV}$. In particular, the spectrum of the low-latitude bubbles is hard and without any apparent fall-off, as compared to the high-latitude bubble spectrum, which shows a sharp cutoff above $100 \mathrm{GeV} \mathrm{[23].}$ As mentioned, the HAWC upper limits [22] do not constrain the low-latitude FB spectrum. The HAWC upper limits derived for the north bubble also do not strictly apply to the south bubble if the south bubble has a gamma-ray morphology and spectrum different from the north bubble. The sample of the high-energy astrophysical neutrinos detected by IceCube has grown to 82 in the meantime (2010-2016) [24], and several more of these events are now from the FB region, as compared to what we had reported in $[18,19]$. Given these developments, it is relevant now to critically review and update the FB hadronic models with relevant constraints.

In this article, we present updated hadronic models for the FB, based on the recent gamma-ray spectral analysis by the Fermi-LAT Collaboration with 6.5 years of data [23], using the high- and low-latitude bubble templates, the latest sample of astrophysical neutrinos detected by IceCube and HAWC constraints at TeV energy gamma ray observations. A new feature in the latest Fermi-LAT data is that the gamma rays from the FB at the high- and low-Galactic latitudes have different spectra. Therefore, we present two hadronic models in the present work fitting those spectra. We take, instead of calculating the proton distribution function fitting the gamma-ray data only (see, e.g., [25]), both the gamma-ray and neutrino data (from this work and from $[18,19,21]$ ) into account to constrain the primary proton spectra. The neutrino data is especially useful to constrain the high-energy cutoff in the primary proton spectrum for the low-latitude case. We discuss details of the latest gamma-ray and neutrino data in Section 2, develop updated hadronic models in Section 3, and discuss possible future constraints from gamma-ray observations in Section 4. We summarize and conclude in Section 5.

\section{Recent Gamma-Ray and Neutrino Data}

The Fermi bubbles, as analyzed with Fermi-LAT data [3,23], were found to have uniform spectra above $10^{\circ}$ in Galactic latitude, with sharp edges. In the most recent update with 6.5 years of Fermi-LAT data, the spectra and morphology of central bubbles have also been derived with the spectral components analysis procedure as in [3]. In this latest analysis, the spectra of low- and high-latitude bubbles are treated separately, but with the assumption that they behave similarly in the energy between 1 and $10 \mathrm{GeV}$. In addition, the center of the bubbles has been found to become brighter as they approach the Galactic plane, which is different from previous models [26] with isotropic emission in the center region. In our study, we adopted the templates derived by the Fermi Collaboration with the region of interest $|\mathrm{b}|<60^{\circ},|1|<45^{\circ}$, as shown in gray contours in Figure 1 , obtaining a solid angle of $\Omega_{F B}=1.04 \mathrm{sr}$, and fit our models with respect to the low- and high-latitude components separately as well.

Recently, the HAWC Collaboration reported their search for very high-energy (VHE) gamma-ray emission from the northern FB region with a latitude above $6^{\circ}$, which corresponds to an FB solid angle of 0.42 sr [22]. No significant excess was found in the analysis, and the $95 \%$ C.L. upper limits consistent with HAWC detection power on the differential flux in four energy bins were obtained as shown in Figure 2. The limits agree with the gamma ray flux above $\sim 2 \mathrm{TeV}$ from the FB regions at high latitudes.

Lately, the IceCube Neutrino Observatory has also updated its high-energy starting event (HESE) search with the neutrino interaction vertex inside the IceCube detector volume and energies above 
$30 \mathrm{TeV}$ using a six-year dataset, which corresponds to a total lifetime of 2078 days. In the full sample, there are 82 events detected, with an expected atmospheric muon background of $25.2 \pm 7.3$ events [24]. Of these events, eight (Nos. 2, 12, 14, 15, 36, 56, 69, and 76) are spatially strongly correlated (the best-fit arrival direction is within the bubble geometry) and six (Nos. 17, 22, 24, 25, 49, 68) are weakly correlated (the median positional error circles overlap with the bubble geometry) with the FB, as seen in Figure 1. Except for Event No. 76, which is a track, all the other events are showers. In particular, within these eight strongly correlated neutrino events, three of them are from the high-latitude northern bubble, four from the high-latitude southern bubble, and one with the highest energy $\sim 1 \mathrm{PeV}$ is from the Galactic Center region. Figure 1 also shows the median positional error for the neutrino arrival directions.

In Figure 2, we show the neutrino spectrum (all three flavors) that we have calculated from the eight events in three energy bins from the FB region using the lifetime and effective areas of IceCube for HESE analysis [24]. There are 3, 4, and 1 event(s), respectively, in the energy ranges $\left(10^{4.2}-10^{4.9}\right)$ $\mathrm{GeV},\left(10^{4.9}-10^{5.6}\right) \mathrm{GeV}$, and $\left(10^{5.6}-10^{6.3}\right) \mathrm{GeV}$. The flux errors on the data points are calculated assuming Poisson statistics.

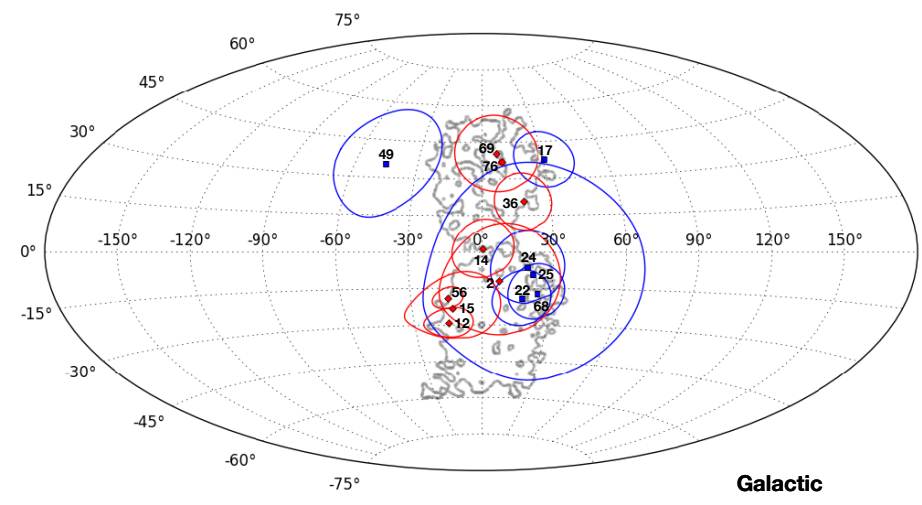

Figure 1. Sky map of the IceCube astrophysical neutrino events [24] that are correlated with the Fermi bubbles in Galactic coordinates. The eight strongly and six weakly correlated events are shown as red diamonds and blue squares, respectively, with their median angular errors and numbers labeled. The Fermi bubble outlines are shown as gray contours.

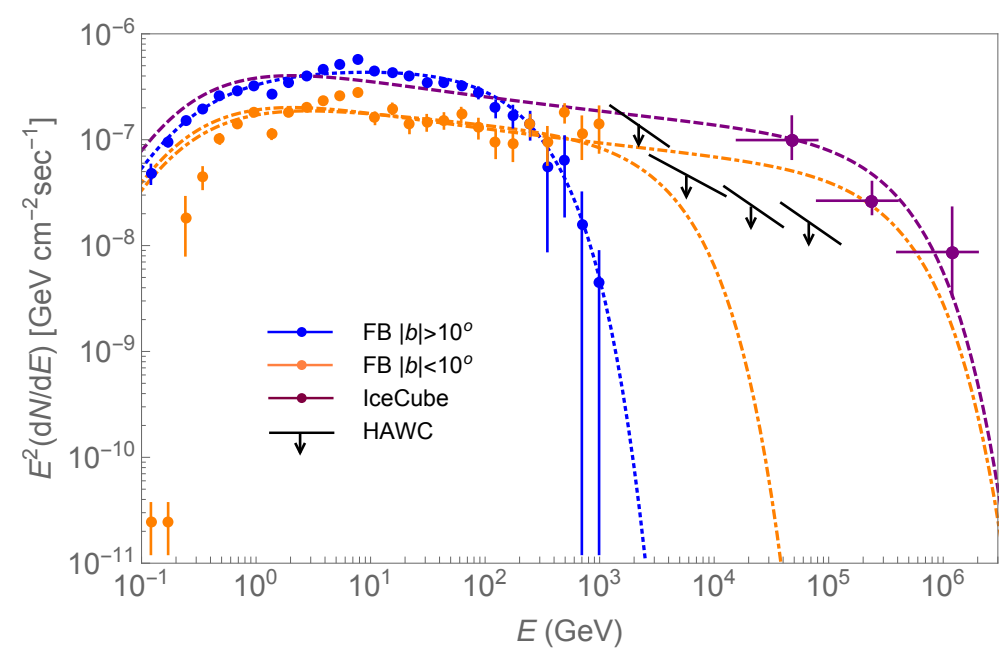

Figure 2. Fermi-LAT spectra from low- and high-latitude regions of the FB [23]. Also shown are HAWC upper limits in the $b>6^{\circ}$ region [22] and neutrino spectra, assumed to be originated from the FB, from eight events [24]. The hadronic models with primary proton spectra $\propto E^{-k} \exp \left(-E / E_{0}\right)$ are shown with lines for $k=2.0, E_{0}=1.6 \mathrm{TeV}$ (blue dotted lines), $k=2.15, E_{0}=30 \mathrm{TeV}$, and $k=2.2, E_{0}=3 \mathrm{PeV}$ (orange dot-dashed lines). The magenta dashed line correspond to the neutrino flux (all three flavors) for the model with $k=2.2, E_{0}=3 \mathrm{PeV}$. 


\section{Hadronic Models}

Prolonged star-formation activity near the Galactic center, which forms a strong bipolar wind above and below the Galactic plane, has been proposed as a plausible mechanism to inflate the FB [10]. Alternately, activity of the Galactic super-massive black hole in the past could also be responsible for the FB origin [2]. In the hadronic models, the gamma rays from the FB are produced primarily from neutral pion decays, which are created by proton-proton $(p p)$ interactions of energetic cosmic rays with dilute gas inside the bubble volumes. Cosmic rays, accelerated to $\sim 10^{15} \mathrm{eV}$ and possibly beyond in supernova remnants, can be carried by the wind to fill the FB volume. Decays of charged pions and kaons, co-produced with neutral pions, by $p p$ interactions guarantee a neutrino flux associated with the gamma-ray flux in the hadronic models.

In our hadronic model formalism $[12,18,21]$, we assume a primary proton spectrum in the form of a power law with an exponentially cutoff such as $N_{p}(E)=N_{0} E^{-k} \exp \left(-E / E_{0}\right)$. Here, $N_{0}, k$, and $E_{0}$ are the normalization factor, the spectral index, and the cutoff energy, respectively. The normalization factor $N_{0}$ is adjusted to fit the gamma-ray spectra by using a particle density of $10^{-2} \mathrm{~cm}^{-3}$ for the gas inside the bubble volumes. The spectral index and the cutoff energy are crucial parameters that can be utilized to constrain the hadronic models from multi-wavelength and multi-messenger observations.

In Figure 2, we show our hadronic model fits for both high-latitude (FB $\left.|b|>10^{\circ}\right)$ and low-latitude (FB $|b|<10^{\circ}$ ) spectra of the FB. The high-latitude spectrum is rather well-fitted with $k=2.0$ and $E_{0}=1.6 \mathrm{TeV}$ (blue dotted lines). This cutoff energy is consistent with the HAWC upper limits in the $\sim 2-200 \mathrm{TeV}$ range, which apply dominantly to the high-latitude part $\left(b>6^{\circ}\right)$ of the northern bubble [22]. We show two model fits (orange dot-dashed lines) for the low-latitude spectra in Figure 2, and they both fit the low-energy $(\leq 10 \mathrm{GeV})$ data rather poorly. The low-energy part of the spectrum is less reliable due to uncertainties in the Galactic emission template at low latitudes and possible contamination with Galactic center emission [23], such as point source detection, in the morphology study of the bubbles, and in the analysis of Galactic interstellar emission. The model with lower cutoff energy has parameters $k=2.15$ and $E_{0}=30 \mathrm{TeV}$, and that with higher cutoff energy has parameters $k=2.2$ and $E_{0}=3 \mathrm{PeV}$. All values of $k$ and $E_{0}$ are consistent with the shock acceleration scenario of cosmic rays in supernova remnants.

The neutrino flux (all three flavors) arising from the hadronic model with $k=2.2$ and $E_{0}=3 \mathrm{PeV}$ is shown in Figure 2 with magenta dashed lines. Although such a high cutoff energy is not required by the Fermi-LAT gamma-ray data alone, an explanation of the neutrino data could be provided with this model. This is also motivated by the hard spectrum of the low-latitude FB without showing any cutoff, unlike the high-latitude spectrum, and by the fact that the HAWC upper limits do not strictly apply in this region. Of course the neutrino events that are strongly correlated with the FB mostly arrive from $|b|<10^{\circ}$ regions in this scenario, which is plausible given large uncertainties in the reconstruction of their directions [24]. Alternatively, it could be that only a fraction of these eight events originate from the FB and that the rest are from a diffuse astrophysical background. We elaborate on this scenario in the following subsection.

\section{Neutrino Events from the FB}

In the recent two-year dataset, the IceCube Observatory detected 28 more HESE neutrinos, and notably all of them are with energies below $200 \mathrm{TeV}$ [24]. Altogether 82 events have been detected so far, and, as Nos. 20 and 55 are coincident with background muons, they are excluded from the analysis. The likelihood fitting was performed in the deposited energy between $60 \mathrm{TeV}$ and $10 \mathrm{PeV}$ by IceCube [24], resulting in a best fit single power law flux of $d N / d E=2.46 \times(E / 100 \mathrm{TeV})^{-2.92} \mathrm{GeV}^{-1} \mathrm{~cm}^{-2} \mathrm{~s}^{-1} \mathrm{sr}^{-1}$ per flavor, which is softer than previous results $[15,27]$ due to the additional events at low energies. Initially, IceCube focused on the higher-energy search around PeV [15], and later the selection threshold of deposited energy was reduced to $\sim 1 \mathrm{TeV}$ [28], where a cosmic ray muon background is dominant. Applying the veto technique as in [28] and the first-level online filters, resulted in an increase in the effective area [24] for both cascade-like and track-like events. 
To estimate the neutrino signal excess from the FB over the 2078-day operation of IceCube, the major backgrounds were atmospheric muons, neutrinos of astrophysical origin, and atmospheric neutrinos in the energy range from $40 \mathrm{TeV}$ to $70 \mathrm{PeV}$. The first case strongly relied on the efficiency of background rejection and the reconstruction technique (see $[15,24]$ for more information). The predicted atmospheric $v_{\mu}+\bar{v}_{\mu}$ flux from [29], averaged over the declination angle of the FB as seen from IceCube, was adopted and extrapolated at high energy. Moreover, the atmospheric $v_{e}+\bar{v}_{e}$ fluxes were greatly suppressed compared to $v_{\mu}+\bar{v}_{\mu}$ by a factor of 14 [30], which was also taken into account. The astrophysical neutrino fluxes from the IceCube best fit adopted here are shown in Figure 3a as a blue line with one-sigma uncertainties (shaded bands), and the hadronic fluxes from the FB region as in Figure 2 are shown as well. All plotted fluxes in Figure 3a are for a solid angle of $\mathrm{FB} \Omega_{\mathrm{FB}}=1.04 \mathrm{sr}$ for all three flavors (combined neutrino and antineutrino).

Since all the neutrinos spatially correlated with FB are cascade events, except for No. 76, we calculated the number of cascade events only with updated HESE effective areas as seen in Figure 3. The number of events in each energy bin $i$ can be calculated as $N_{i}=\int_{E_{\mathrm{i}, \min }}^{E_{\mathrm{i}_{\text {max }}}}(d N / d E) A_{\mathrm{eff}} T_{\exp } \Omega_{\mathrm{FB}}$, where $A_{\text {eff }}$ and $T_{\text {exp }}$ are the $v_{e}$ or $v_{\tau}$ effective area and exposure time (2078 days), respectively. These event distributions are plotted in Figure 3. We expected a total of 8.8 and 7.9 cascade-like events from the backgrounds (atmospheric + astrophysical) and FB, respectively, in the first five energy bins. Given the harder spectrum of the FB flux than the diffuse astrophysical flux, the signature of the FB was more prominent in the $\sim 100 \mathrm{TeV}-1 \mathrm{PeV}$ range. Within one sigma uncertainty, the number of background events can be reduced to 5.5. The estimation agrees with the IceCube observation, where 8 (6) events are strongly (weakly) correlated with the FB. One note of caution, however, is that the diffuse flux fit is based on all events, including a plausible contribution from the FB.

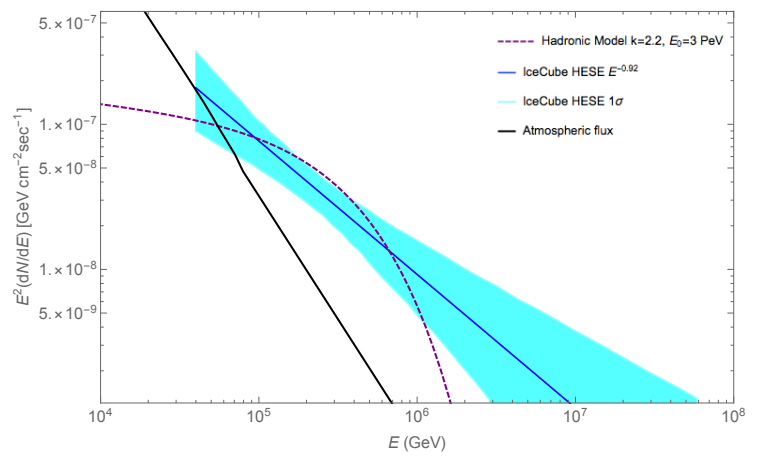

(a)

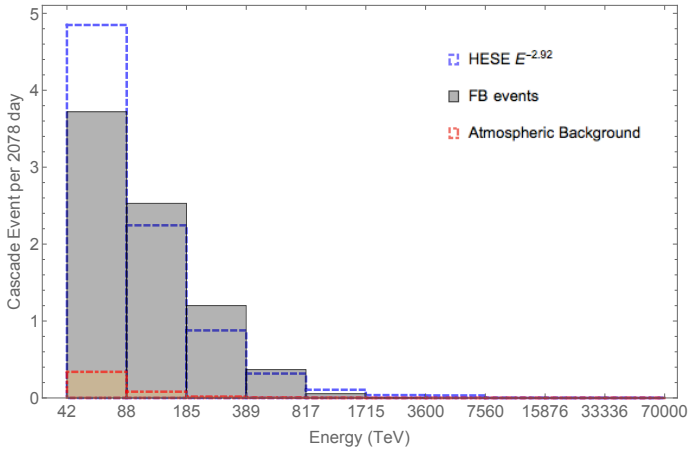

(b)

Figure 3. (a) Neutrino fluxes. Best-fit astrophysical neutrino fluxes with a single power law of $E^{-0.92}$ and one sigma uncertainties as represented by the blue shading. The black line represents the atmospheric neutrino fluxes, and the magenta line is the hadronic flux in Figure 2. (b) Neutrino event rate. The expected number of neutrino events from the FB as well as the astrophysical and atmospheric backgrounds are represented by gray bars as well as red and white (dashed blue edge) bars, respectively.

Detection of track-like neutrino events, which have $\leq 1^{\circ}$ angular resolution, by the upcoming KM3NeT Neutrino Telescope located in the northern hemisphere [31] will be helpful to separate the FB from other possible Galactic sources, as well as complimentary to the IceCube data dominated by shower-like events.

\section{Future VHE Gamma-Ray Constraints on Hadronic Models}

The HAWC observatory found no significant excess towards the FB using the data between 27 November 2014 to 11 February 2016, resulting in 90\% upper limits on the high-latitude northern 
bubble with $\mathrm{b}>6^{\circ}$ at energies between 1 and $100 \mathrm{TeV}$ [22]. These upper limits agree with our hadronic emission model of the high-latitude FB, which is strongly suppressed beyond $\sim 1 \mathrm{TeV}$ (see Figure 2). The spectrum of the low-latitude FB is harder and without any apparent cutoff in the energy range of the Fermi-LAT. Therefore, both our nominal model with parameters $k=2.15$ and $E_{0}=30 \mathrm{TeV}$ and the neutrino-inspired model with parameters $k=2.2$ and $E_{0}=3 \mathrm{PeV}$ for the low-latitude spectrum are potential targets for future VHE observations. We expect that the HAWC collaboration will further analyze data from the FB in the future. We expect that such data will include the sensitivity at lower energies and a larger field of view that includes the Galactic Center region will be employed. This will provide more stringent constraints on both the spectra at low- and high-latitude bubbles, the full image of non-uniform intensity, and the shape of the central region.

On the other hand, it is hoped that the Cherenkov Telescope Array, the next generation of ground-based VHE observations [32], from observatories with a northern (La Palma) and a southern (Chile) site, will be able to observe both bubbles. A detailed strategy needs to be developed about detecting different parts of the FB and better controlling the background. In Figure 4 , we show the differential sensitivity of the CTA-South for $50 \mathrm{~h}$ observation of a point source [32] for illustration purposes. Moreover, the Large High Altitude Air Shower Observatory (LHAASO), being built at a $4410 \mathrm{~m}$ altitude in the Sichuan province of China and detecting cosmic rays and gamma rays in the energy range of $100 \mathrm{GeV}-1 \mathrm{PeV}$ [33], will be complementary to the HAWC and CTA. With a high duty cycle and wide field of view, $\approx \pi \mathrm{sr}$, the LHAASO will be able to observe the entire northern bubble with a solid angle of 0.45 sr. We show the differential sensitivity of the LHAASO in Figure 4 for one year of observation of a Crab-like point source [33], again for illustration purpose. A detailed spectral and morphological study with simulations will be required to calculate the sensitivities of the CTA and HAWC to the FB.

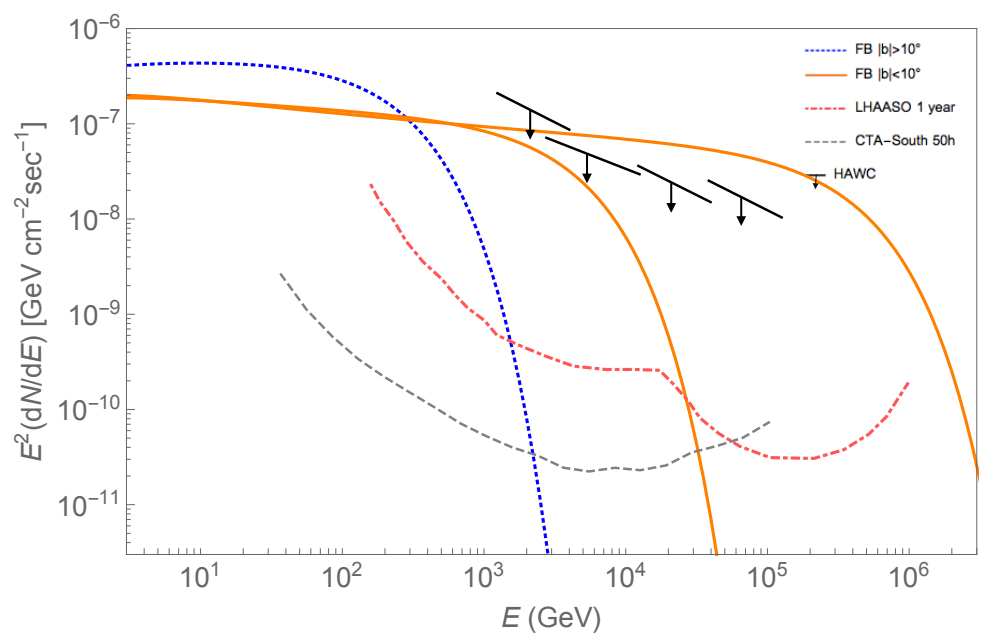

Figure 4. FB spectra from the low- and high-latitude regions and HAWC upper limits as shown in Figure 2. The differential point source flux sensitivities of the CTA southern site [32] with $50 \mathrm{~h}$ of observation (gray dashed curve) and of the LHAASO [33] with one year of observation (red dot-dashed curve) are shown with the FB flux models for illustration purposes.

\section{Summary and Outlook}

In this study, hadronic modeling of the $0.1 \mathrm{GeV}-1 \mathrm{TeV}$ gamma-ray emission from the FB was updated based on new data from the Fermi-LAT, upper limits from the HAWC, and an expanded astrophysical neutrino sample from the IceCube. The latest analysis of Fermi-LAT data prefers a spectrum with a cutoff above $1 \mathrm{TeV}$ in the high Galactic latitude $\left(|b|>10^{\circ}\right)$ region of the FB, while a hard spectrum without any apparent cutoff in the low Galactic latitude $\left(|b|<10^{\circ}\right)$ region of the FB [23]. Combined with the latest IceCube astrophysical neutrino data [24], our updated hadronic model can 
be extended to the $1 \mathrm{PeV}$ range for the low-latitude $\mathrm{FB}$ emission and is not directly constrained by the HAWC upper limits derived from observations of the northern bubble at high-latitude $\left(b>6^{\circ}\right)$ [22]. Our updated hadronic model for the high-latitude FB spectrum is consistent with the HAWC upper limits.

The observation of high-energy neutrinos from the FB would strongly support the hadronic origin of gamma rays. The detection of several more astrophysical neutrino events by IceCube from the direction of the FB in the latest dataset is therefore intriguing. Our estimated FB neutrino flux based on these events is harder than a diffuse astrophysical flux estimate by the IceCube collaboration, although it is not possible to distinguish them using the current data set. Future observation of neutrinos by KM3NeT [31] from the FB region can shed insights, especially if many track-like events are detected with good angular resolution. Observations of VHE gamma rays from the FB with the upcoming CTA [32] and LHAASO [33], combined with deeper observations by HAWC, will be crucial to probe the spectra of the $\mathrm{FB}$ in the 1-100 $\mathrm{TeV}$ range, which ties with the lower energy range of the IceCube neutrino events. The detection of a cutoff in this energy range for the low-latitude FB spectrum can strongly constrain hadronic models that can explain the IceCube neutrino data and critically test the $\mathrm{FB}$ as a multi-messenger source.

Acknowledgments: We thank D. Malyshev for providing the latest Fermi-LAT spectral data. S.R. acknowledges support from the National Research Foundation (South Africa) with Grant No: 111749 (CPRR). L.Y. acknowledges the Slovenia Research Agency grant number Z1-8139 (B).

Author Contributions: S.R. and L.Y. conceived and designed the investigation, analyzed the data, performed the modeling, and wrote the paper.

Conflicts of Interest: The authors declare no conflict of interest. The funding sponsors had no role in the design of the study; in the collection, analyses, or interpretation of data; in the writing of the manuscript; or in the decision to publish the results.

\section{References}

1. Dobler, G.; Finkbeiner, D.P.; Cholis, I.; Slatyer, T.R.; Weiner, N. The Fermi Haze: A Gamma-Ray Counterpart to the Microwave Haze. Astrophys. J. 2010, 717, 825. [CrossRef]

2. Su, M.; Slatyer, T.R.; Finkbeiner, D.P. Giant Gamma-ray Bubbles from Fermi-LAT: AGN Activity or Bipolar Galactic Wind? Astrophys. J. 2010, 724, 1044. [CrossRef]

3. Ackermann, M.; Albert, A.; Atwood, W.B.; Baldini, L.; Ballet, J.; Barbiellini, G.; Bastieri, D.; Bellazzini, R.; Bissaldi, E.; Blandford, R.D.; et al. The Spectrum and Morphology of the Fermi Bubbles. Astrophys. J. 2014, 793, 64. [CrossRef]

4. Narayanan, S.A.; Slatyer, T.R. A Latitude-Dependent Analysis of the Leptonic Hypothesis for the Fermi Bubbles. Mon. Not. R. Astron. Soc. 2017, 468, 3051-3070. [CrossRef]

5. Finkbeiner, D.P. Microwave ism emission observed by wmap. Astrophys. J. 2004, 614, 186-193. [CrossRef]

6. Aghanim, N.; Arnaud, M.; Ashdown, M.; Atrio-Barandela, F.; Aumont, J.; Baccigalupi, C.; Balbi, A.; Banday, A.J.; Barreiro, R.B.; Bartlett, J.G.; et al. Planck Intermediate Results. IX. Detection of the Galactic haze with Planck. Astron. Astrophys. 2013, 554, A139. [CrossRef]

7. Snowden, S.L.; Egger, R.; Freyberg, M.J.; McCammon, D.; Plucinsky, P.P.; Sanders, W.T.; Schmitt, J.H.M.M.; Truemper, J.; Voges, W. ROSAT Survey Diffuse X-Ray Background Maps. II. Astrophys. J. 1997, 485, 125. [CrossRef]

8. Tahara, M.; Kataoka, J.; Takeuchi, Y.; Totani, T.; Sofue, Y.; Hiraga, J.S.; Tsunemi, H.; Inoue, Y.; Kimura, M.; Cheung, C.C.; et al. Suzaku X-ray Observations of the Fermi Bubbles: Northernmost Cap and Southeast Claw Discovered with MAXI-SSC. Astrophys. J. 2015, 802, 91. [CrossRef]

9. Carretti, E.; Crocker, R.M.; Staveley-Smith, L.; Haverkorn, M.; Purcell, C.; Gaensler, B.M.; Bernardi, G.; Kesteven, M.J.; Poppi, S. Giant Magnetized Outflows from the Centre of the Milky Way. Nature 2013, 493, 66. [CrossRef] [PubMed] 
10. Crocker, R.M.; Aharonian, F. The Fermi Bubbles: Giant, Multi-Billion-Year-Old Reservoirs of Galactic Center Cosmic Rays. Phys. Rev. Lett. 2011, 106, 101102. [CrossRef] [PubMed]

11. Crocker, R.M.; Bicknell, G.V.; Taylor, A.M.; Carretti, E. A Unified Model of the Fermi Bubbles, Microwave Haze, and Polarized Radio Lobes: Reverse Shocks in the Galactic Center's Giant Outflows. Astrophys. J. 2015, 808, 107. [CrossRef]

12. Lunardini, C.; Razzaque, S. High Energy Neutrinos from the Fermi Bubbles. Phys. Rev. Lett. 2012, 108, 221102. [CrossRef] [PubMed]

13. Mertsch, P.; Sarkar, S. Fermi gamma-ray 'bubbles' from stochastic acceleration of electrons. Phys. Rev. Lett. 2011, 107, 091101. [CrossRef] [PubMed]

14. Cheng, K.S.; Chernyshov, D.O.; Dogiel, V.A.; Ko, C.-M.; Ip, W.-H. Origin of the Fermi Bubble. Astrophys. J. 2011, 731, L17. [CrossRef]

15. Aartsen, M.G.; Abbasi, R.; Abdou, Y.; Ackermann, M.; Adams, J.; Aguilar, J.A.; Ahlers, M.; Altmann, D.; Auffenberg, J.; Bai, X.; et al. Evidence for High-Energy Extraterrestrial Neutrinos at the IceCube Detector. Science 2013, 342, 1242856. [CrossRef] [PubMed]

16. Razzaque, S. The Galactic Center Origin of a Subset of IceCube Neutrino Events. Phys. Rev. D 2013, 88, 081302. [CrossRef]

17. Ahlers, M.; Murase, K. Probing the Galactic Origin of the IceCube Excess with Gamma-Rays. Phys. Rev. D 2014, 90, 023010. [CrossRef]

18. Lunardini, C.; Razzaque, S.; Theodoseau, K.T.; Yang, L. Neutrino Events at IceCube and the Fermi Bubbles. Phys. Rev. D 2014, 90, 023016. [CrossRef]

19. Lunardini, C.; Razzaque, S.; Yang, L. Neutrino events at IceCube and the Fermi bubbles. arXiv, 2014, arXiv:1412.6240.

20. Fang, K.; Su, M.; Linden, T.; Murase, K. IceCube and HAWC constraints on very-high-energy emission from the Fermi bubbles. Phys. Rev. D 2017, 96, 123007. [CrossRef]

21. Lunardini, C.; Razzaque, S.; Yang, L. Multimessenger study of the Fermi bubbles: Very high energy gamma rays and neutrinos. Phys. Rev. D 2015, 92, 021301. [CrossRef]

22. Abeysekara, A.U.; Albert, A.; Alfaro, R.; Alvarez, C.; Alvarez, J.D.; Arceo, R.; Arteaga-Velázquez, J.C.; Solares, H.A.; Barber, A.S.; Bautista-Elivar, N.; et al. Search for Very High-energy Gamma Rays from the Northern Fermi Bubble Region with HAWC. Astrophys. J. 2017, 842, 85. [CrossRef]

23. Ackermann, M.; Ajello, M.; Albert, A.; Atwood, W.B.; Baldini, L.; Ballet, J.; Barbiellini, G.; Bastieri, D.; Bellazzini, R.; Bissaldi, E.; et al. The Fermi Galactic Center GeV Excess and Implications for Dark Matter. Astrophys. J. 2017, 840, 43. [CrossRef]

24. Aartsen, M.G.; Ackermann, M.; Adams, J.; Aguilar, J.A.; Ahlers, M.; Ahrens, M.; Samarai, I.A.; Altmann, D.; Andeen, K.; Anderson, T.; et al. The IceCube Neutrino Observatory-Contributions to ICRC 2017 Part II: Properties of the Atmospheric and Astrophysical Neutrino Flux. arXiv, 2017, arXiv:1710.01191.

25. Fujita, Y.; Ohira, Y.; Yamazaki, R. A Hadronic-Leptonic Model for the Fermi Bubbles: Cosmic-Rays in the Galactic Halo and Radio Emission. Astrophys. J. 2014, 789, 67. [CrossRef]

26. Ajello, M.; Albert, A.; Atwood, W.B.; Barbiellini, G.; Bastieri, D.; Bechtol, K.; Bellazzini, R.; Bissaldi, E.; Blandford, R.D.; Bloom, E.D.; et al. Fermi-LAT Observations of High-Energy $\gamma$-Ray Emission Toward the Galactic Center. Astrophys. J. 2016, 819, 44. [CrossRef]

27. Kopper, C.; Giang, W.; Kurahashi, N. Observation of Astrophysical Neutrinos in Four Years of IceCube Data. PoS ICRC 2015, 30, 1081.

28. Aartsen, M.G.; Ackermann, M.; Adams, J.; Aguilar, J.A.; Ahlers, M.; Ahrens, M.; Altmann, D.; Anderson, T.; Arguelles, C.; Arlen, T.C.; et al. Atmospheric and astrophysical neutrinos above $1 \mathrm{TeV}$ interacting in IceCube. Phys. Rev. D 2015, 91, 022001. [CrossRef]

29. Honda, M.; Kajita, T.; Kasahara, K.; Midorikawa, S.; Sanuki, T. Calculation of atmospheric neutrino flux using the interaction model calibrated with atmospheric muon data. Phys. Rev. D 2007, 75, 043006. [CrossRef]

30. Sinegovskaya, T.S.; Ogorodnikova, E.V.; Sinegovsky, S.I. High-energy fluxes of atmospheric neutrinos. arXiv, 2013, arXiv:1306.5907.

31. Adrián-Martínez, S.; Ageron, M.; Aharonian, F.; Aiello, S.; Albert, A.; Ameli, F.; Anassontzis, E.; Andre, M.; Androulakis, G.; Anghinolfi, M.; et al. Letter of intent for KM3NeT 2.0. J. Phys. G 2016, 43, 084001. [CrossRef] 
32. Consortium, T.C.T.A.; Acharya, B.S.; Agudo, I.; Samarai, I.A.; Alfaro, R.; Alfaro, J.; Alispach, C.; Batista, R.A.; Amans, J.P.; Amato, E.; et al. Science with the Cherenkov Telescope Array. arXiv, 2017, arXiv:1709.07997.

33. Di Sciascio, G.; LHAASO Collaboration. The LHAASO experiment: from Gamma-Ray Astronomy to Cosmic Rays. Nucl. Part. Phys. Proc. 2016, 279-281, 166-173. [CrossRef]

(C) 2018 by the authors. Licensee MDPI, Basel, Switzerland. This article is an open access article distributed under the terms and conditions of the Creative Commons Attribution (CC BY) license (http:/ / creativecommons.org/licenses/by/4.0/). 